\title{
Inheritance of a mitochondrial DNA defect and impaired glucose tolerance in BHE/Cdb rats
}

\author{
C.E. Mathews ${ }^{1}$, R.A. McGraw ${ }^{2}$, R. Dean ${ }^{3}$, C.D. Berdanier ${ }^{1}$ \\ ${ }^{1}$ Department of Foods and Nutrition, University of Georgia, Athens, Georgia, USA \\ ${ }^{2}$ Department of Physiology and Pharmacology, College of Veterinary Medicine, University of Georgia, Athens, Georgia, USA \\ ${ }^{3}$ Department of Animal Science, University of Georgia, Athens, Georgia, USA
}

\begin{abstract}
Summary As they age, BHE/Cdb rats develop impaired glucose tolerance. We hypothesized that this intolerance is associated with a previously reported base substitution in the mitochondrial genome. A new screening test was devised to identify animals with the mutation. These animals were bred to animals without the mutation. The progeny were then tested for the presence of the mutation and their glucose tolerance at 100 and 300 days of age. Phenotype
\end{abstract}

and genotype were found to be closely linked and we conclude that the mutation in the mitochondrial ATPase 6 gene explains the age related impaired glucose tolerance in $\mathrm{BHE} / \mathrm{Cdb}$ rats. [Diabetologia (1999) 42: 35-40]

Keywords BHE/Cdb Rat, mitochondrial diabetes, point mutation, gene screen, allele specific amplification, competitive PCR, maternal inheritance.
The BHE/Cdb rat strain is an inbred strain that develops impaired glucose tolerance at maturity. The diabetic state is preceded by defects in metabolism that progress with age. Environmental factors, such as diet, affect the time frame for the phenotypic expression of the genotype [1]. Feeding sugar rich and fat rich diets hastens the appearance of impaired glucose tolerance and its complications. The diabetic phenotype has been attributed to point mutations (bp 8204, 8289) in the mtDNA gene for $\mathrm{F}_{\mathrm{o}}$ ATPase subunit 6 [2] and Herrnstad, (personal communication) because these rats show impaired mitochondrial

Received: 12 December 1997 and in final revised form: 31 July 1998

Corresponding author: C.D. Berdanier, Ph.D., Department of Foods and Nutrition, The University of Georgia, Athens, GA 30602-3622, USA

A preliminary report of this work was reported at the International Workshop on Animal Diabetes held in Copenhagen, July 14-17, 1997.

Abbreviations: SD, Sprague Dawley; PCR, polymerase chain reaction; ANOVA, analysis of variance; $\mathrm{BHE} / \mathrm{Cdb}$, progeny of two $\mathrm{BHE} / \mathrm{Cdb}$ rats; SD, progeny of two SD rats; $\mathrm{BX}$, progeny of $\mathrm{BHE} / \mathrm{Cdb}$ mother and SD father; SX, progeny of SD mother and $\mathrm{BHE} / \mathrm{Cdb}$ father. function, particularly in the control of oxidative phosphorylation [3-5]. No evidence of a point mutation in the tRNA ${ }^{\text {LEU(UUR) }}$ has been found nor have we found evidence of muscle or nerve pathology. This rat strain shows a reduction in ATP synthesis efficiency, mitochondrial coupling and $\beta$-cell ATP content. A decline in glucose stimulated insulin release also occurs with age [6]. Glucose stimulated insulin release falls from $89.5 \pm 10.2 \mathrm{pmol} / 1$ to $46.3 \pm 22.7 \mathrm{pmol} / 1$ at $30 \mathrm{~s}$ post glucose injection and from $97.6 \pm 21.4 \mathrm{pmol} / \mathrm{l}$ to $74.6 \pm 11.0 \mathrm{pmol} / \mathrm{l}$ at $60 \mathrm{~s}$ as the animals age from 50 to 300 days of age. Corresponding values in Sprague Dawley rats (SD) at 300 days of age were $74.6 \pm$ $38.2 \mathrm{pmol} / \mathrm{l}$ and $110.7 \pm 12.2 \mathrm{pmol} / \mathrm{l}$. The values from $\mathrm{BHE} / \mathrm{Cdb}$ rats were roughly $30 \%$ less than those of the SD rats. Islets isolated from these rats release $65 \%$ less insulin than islets from control rats of the same age when stimulated by the addition of glucose to the incubation medium. There was, however, only a small difference in islet insulin content $(0.021 \pm 0.002 \mathrm{pmol} / \mathrm{g}, 0.020 \pm 0.009 \mathrm{pmol} / \mathrm{g}$ dry tissue, $\mathrm{SD}$ vs $\mathrm{BHE} / \mathrm{Cdb}$ ) at 50 days of age.

The $\mathrm{F}_{\mathrm{o}}$ ATPase subunit 6 (also called subunit a) mutation [2] could account for the age related decline in ATP synthesis efficiency and subsequent alterations in glucose metabolism. A maternal mode of in- 
Table 1. Primers for the gene screen

\begin{tabular}{|c|c|c|c|c|c|c|}
\hline SD & HS & & ССТАССАСТС & AGCTATCTAT & $\mathrm{A} \underline{\mathrm{G}^{1}}$ & $(22)^{2}$ \\
\hline SD & LS & & TTTTTAGTTT & GTGTCGGAA $\underline{A}$ & & (20) \\
\hline $\begin{array}{l}\text { BHE } \\
\text { BHE }\end{array}$ & $\begin{array}{l}\text { HS } \\
\text { LS }\end{array}$ & $\begin{array}{l}++++++++++ \\
\text { GCCCGGATCC } \\
\text { GCCCGAATTC }\end{array}$ & $\begin{array}{l}\text { ССТАССАСТC } \\
\text { TTTTTAGTTT }\end{array}$ & $\begin{array}{l}\text { AGCTATCTAT } \\
\text { GTGTCGGAAG }\end{array}$ & $\mathrm{A} \underline{\mathrm{A}}$ & $\begin{array}{l}(32) \\
(30)\end{array}$ \\
\hline
\end{tabular}

Primer Names: SD is the wild type or the Sprague Dawley genotype. $\mathrm{BHE}$ is the mutated or $\mathrm{BHE} / \mathrm{Cdb}$ genotype. $\mathrm{HS}=$ Heavy Strand. LS $=$ Light Strand.

${ }^{1}$ The underlined nucleotide denotes the base difference (A for

G) at position 8204 of the heavy strand and is at the 3' end of

heritance of the Type II (non-insulin-dependent) diabetes mellitus trait was suspected based on breeding records. If the impaired glucose tolerance was due to the mitochondrial defect, both the impaired glucose tolerance and the DNA mutation should follow a maternal inheritance pattern. To test this hypothesis a cross breeding study of glucose tolerance and mt ATPase sequence was conducted using, as parents, animals with or without the mutation. Parents and progeny were genotypically screened. To determine the genotype it was necessary to develop a quick and reliable test to determine if the point mutation was present. Thus the second goal of this work was to develop such a screen. This paper reports the results of this work directed towards linking the genotype of the $\mathrm{BHE} / \mathrm{Cdb}$ rats to its impaired glucose tolerance phenotype.

\section{Materials and methods}

Development of the polymerase chain reaction (PCR)-based screen and determination of genotype. Sprague Dawley rats were purchased from Harlan Sprague Dawley (Madison, Wis., USA) and BHE/Cdb rats were obtained from the (University of Georgia (USA) Athens GA) Breeding Colony. Sprague Dawley and $\mathrm{BHE} / \mathrm{Cdb}$ rats were fed a standard stock diet and killed at 50 days of age. Muscle, pancreas, liver, tip of tail and kidneys were harvested to determine the universality of the mitochondrial mutation. These tissues were used for DNA isolation using methods described previously [7]. The tail tip DNA was extracted as follows: the tail tip, Proteinase $\mathrm{K}$ and PBND were added to individual $0.6 \mathrm{ml}$ Eppendorf tubes. This was left shaking overnight at $55^{\circ}$. The reaction was then heated to $95^{\circ}$ for $10 \mathrm{~min}$ with occasional mixing. The tubes were cooled to room temperature and then diluted with distilled water. This extract was then used for DNA isolation using the same methods used for the other tissues [2].

The PCR screen used two different sets of primers to detect mutated animals from wild type (Table 1 ). The PCR products were sequenced and found identical to those reported earlier [2]. Primers were synthesized by Genemed Biotechnologies, Inc. (San Francisco, Calif., USA). Allele specific extension primers were constructed such that their 3 ' ends were complementary to the site of the mutational difference between the normal and mutated DNA. These sequence differences allowed the primers in the wild type set to be designed with different bases on the $3^{\prime}$ end than the analogous primer set for the BHE/Cdb type. This floppy 3 ' end concept permitted in a this sequence. The corresponding light strand also begins with a base difference in the reverse ( $\mathrm{G}$ for $\mathrm{A})$.

+- Denotes the added restriction sites for the differentiation in length.

${ }^{2}$ Length of the primer

competitive PCR, the primers with only the correct 3 ' nucleotide to anneal and extend. Thus, we developed a competitive allele specific amplification test that enabled us to segregate the two genotypes.

The primers were synthesized so that the products of the two sets of primers were of different lengths yet the primers still had calculated annealing temperatures that were approximately equal. This was accomplished with the addition of restriction sites onto the $5^{\prime}$ ends of the primers specific for the mutated genotype. Restriction sites were added to the primers should multiple bands be observed suggesting heteroplasmy. The addition of the restriction site to the primer (the primers were synthesized for 2 point mutations, one that was upstream and one that was downstream) could then facilitate the identification of the mutated genome. The PCR product could be cut with the two restriction enzymes in two separate restriction digestions and the result would tell which point mutation was harboured heteroplasmically. Since only one band for each strain was found, however, this additional step was not needed. The reactions were carried out in a Perkin-Elmer Gene Amp PCR system 2400 (Perkin-Elmer, Foster City, Calif., USA). The volume of the reaction was $50 \mu \mathrm{l}$ which contained: $0.25 \mu \mathrm{g}$ each of all 4 primers, $50 \mathrm{mmol} / \mathrm{l} \mathrm{KCl}, 10 \mathrm{mmol} / \mathrm{l}$ Tris- $\mathrm{HCl}, \mathrm{pH}$ $8.3,1 \mathrm{mmol} / 1 \mathrm{MgCl}_{2}, 0.1 \%$ Triton-X, 2.5 units Taq Polymerase, $1.25 \mu \mathrm{mol} / 1$ of each dNTP and $1 \mu \mathrm{g}$ of template DNA. All reactions were "hot start". The conditions for the PCR were: $94^{\circ}$ for $6 \mathrm{~min}$, then 25 cycles of: $94^{\circ}$ for $30 \mathrm{~s}, 55^{\circ}$ for $30 \mathrm{~s}$ and $72^{\circ}$ for $3 \mathrm{~min}$ and then held at $4^{\circ}$. The genotype was assigned when the results of the competitive PCRs were electrophoresed. An aliquot $(9 \mu \mathrm{l})$ from each of the completed PCR reactions was mixed with $1 \mu \mathrm{l}$ of dye and loaded on a $4 \%$ agarose gel. The location of the band determined the genotype since the smaller product would migrate further on the gel. The $\mathrm{BHE} / \mathrm{Cdb}$ (mutated) genotype yielded a band of 113 base pairs in length while the wild type genotype yielded a band 93 base pairs in length. A 1:1 mixture of the BHE/Cdb and SD DNA was similarly prepared and the PCR products were used to produce a ladder. Each time the screen was used to determine genotype both negative and positive controls were run. Negative controls consisted of the essential PCR components with the exception of template DNA. Template DNA in the heteroplasmic positive control was synthesized in PCR reactions which used a very low annealing temperature $\left(35^{\circ}\right)$ for the first 5 cycles and an annealing temperature $55^{\circ}$ for the following 20 cycles. The separate reactions were run and using either the primer pair SD HS and BHE LS or the pair of BHE HS and SD LS. This yielded PCR products which contained either wild type at 8204 and mutated at 8251 or mutated at 8204 and wild type at 8251 . Products of these two PCR reactions were run on $4 \%$ agarose gels, the $103 \mathrm{bp}$ band excised and the products electroeluted and purified using QIAquick purification kit (QIAGEN, Hilden, Germany). Positive reactions contained $0.25 \mathrm{mg}$ each of all 4 primers, $50 \mathrm{mmol} / \mathrm{l} \mathrm{KCl,} 10 \mathrm{mmol} / \mathrm{l}$ Tris- 
Table 2. Cross Breeding Design

\begin{tabular}{lll}
\hline $\begin{array}{l}\text { Female Parents } \\
\text { Male Parents }\end{array}$ & $\begin{array}{l}\text { BHE/Cdb Female } \\
\text { (Mutant) }\end{array}$ & $\begin{array}{l}\text { SD Female } \\
\text { (Wild Type) }\end{array}$ \\
\hline $\begin{array}{l}\text { BHE/Cdb Male } \\
\text { (Mutant) }\end{array}$ & BHE/Cdb & SX \\
$\begin{array}{l}\text { SD Male } \\
\text { (Wild Type) }\end{array}$ & BX & Sprague Dawley \\
\hline
\end{tabular}

$\mathrm{HCl}, \mathrm{pH} 8.3,1 \mathrm{mmol} / \mathrm{l} \mathrm{MgCl}_{2}, 0.1 \%$ Triton-X, 2.5 units Taq Polymerase, $1.25 \mathrm{mmol} / \mathrm{l}$ of each $\mathrm{dNTP}$ and $1 \mathrm{mg}$ of either $\mathrm{BHE}$, or SD, PCR product (SD HS/BHE LS) or (BHE HS/ SD LS). These reactions yielded 113, 93, 103 (cleavable to 98 with Eco R1) and 103 (cleavable to 98 with Bam H1) respectively. Screening was done on all parents and progeny using the tail tip.

Determination of phenotype. Female and male $\mathrm{BHE} / \mathrm{Cdb}$ and Sprague Dawley rats were screened for the presence of the mutation before breeding. They were bred and their progeny were also screened. Using the above methods, DNA was extracted from tissue of the tip of the tail and PCRs were run as described above. The tail tip tissue was harvested asceptically. Table 2 shows the breeding scheme used. Animals were bred at 150 days of age. Six breeding pairs for each cross ( 24 pairs, 48 rats) were used. The progeny were labelled BHE/Cdb or SD indicating that both parents were of the same strain and $\mathrm{BX}$ where the mother was from the BHE/Cdb strain and the father was of the Sprague-Dawley strain. The SX designation referred to progeny of a Sprague Dawley mother and BHE/Cdb father. They were tested for glucose intolerance at 100 and 300 days of age. Animals were starved for 14-16 h overnight prior to the test. A fasting blood sample was obtained from the cut tip of the tail. Each rat was gavaged with a $25 \%$ glucose solution at a dose of $0.1 \mathrm{cc} / 100 \mathrm{~g}$ body weight $(1 \mathrm{~g}$ glucose $/ \mathrm{kg}$ body weight). Blood samples were collected at 30, 60 and 120 min post gavage into ice cold $10 \times 75 \mathrm{~mm}$ glass test tubes. Samples were stored on ice until all collections were complete. Serum was collected after centrifugation at $3500 \mathrm{rpm}, 4^{\circ} \mathrm{C}$ for $20 \mathrm{~min}$ and used to determine glucose by glucose oxidase (Sigma, kit no. 510, Sigma Chemical Co., St. Louis, Mo., USA).

Statistical analysis. Statistical significance was determined using Super analysis of variance (ANOVA) for the Macintosh (Abacus Concepts, Berkeley, Calif., USA) using a one way ANOVA and a Fisher's least significant difference test (LSD) to determine $p$ values.

Fig. 1. Determination of homoplasmy in BHE/Cdb and Sprague Dawley rats. Ladder is in lane 1 and the Bands are 93, 100 and 113 base pairs. The ladder is a 1:1 mix of SD and BHE/ $\mathrm{Cdb}$ PCR products. $\mathrm{BHE}=\mathrm{PCR}$ products of the $\mathrm{BHE} / \mathrm{Cdb}$ strain. $\mathrm{SD}=\mathrm{PCR}$ products of the Sprague Dawley Strain

\section{Results}

Screening of the animals for genotype. Figure 1 shows that all products from the competitive allele specific amplification assay (ASA) using tissue from the $\mathrm{BHE} / \mathrm{Cdb}$ rats were 113 pairs in length and that the products of the all of the Sprague Dawley (SD) rat competitive allele specific amplifications were 93 base pairs in length. Also shown is the universality of the mitochondrial mutation. All the tissues tested were homeoplasmic for the strain specific ATPase 6 sequence. There was no evidence of heteroplasmy. A total of 12 rats (6/strain) were used for this phase of the work.

Figure 2 shows PCR products from the competitive allele specific amplification assay using tissues from BHE/Cdb and Sprague Dawley parents. As in Figure 1 , the $\mathrm{BHE} / \mathrm{Cdb}$ rats have a product of 113 base pairs and the Sprague Dawley rats have a product of 93 base pairs in length. The genotypes of the offspring are shown in Figure 3. As in Figure 2, the BHE/Cdb and $\mathrm{BX}$ rats have PCR products of 113 base pairs and the Sprague Dawley and SX rats have PCR products of only 93 base pairs in length. This shows that the genotype is maternally inherited as expected. In this part of the study all 48 parents and their collective progeny were screened using the tail tip tissue.

Determination of phenotype. In the fasting state all the animals were normoglycaemic. The curves for glucose tolerance in $\mathrm{BHE} / \mathrm{Cdb}$ and $\mathrm{BX}$ rats displayed larger areas under the curve than the SD rats (Table 3) with values at 30,60 and 120 min exceeding those of SD rats. The SX rats were no different from their SD controls at 30, 60 and 120 min (Fig. 4). Note that the blood glucose values did not return to the prechallenge concentrations in the $\mathrm{BHE} / \mathrm{Cdb}$ and $\mathrm{BX}$ rats but the values for the $\mathrm{SD}$ and $\mathrm{SX}$ rats did. Note also that the 300-day-old BHE/Cdb and BX had an essentially flat post glucose value at 300 days of age whereas the 300 day old SD and SX rats had 120-min glucose values that were similar to their prechallenge values. These results show that the impaired glucose tolerance trait is maternally inherited.

\section{Discussion}

The results of the present work clearly show that the mitochondrial genomic trait of a base substitution in the ATPase 6 (subunit a of $F_{o}$ ATPase) gene associ-

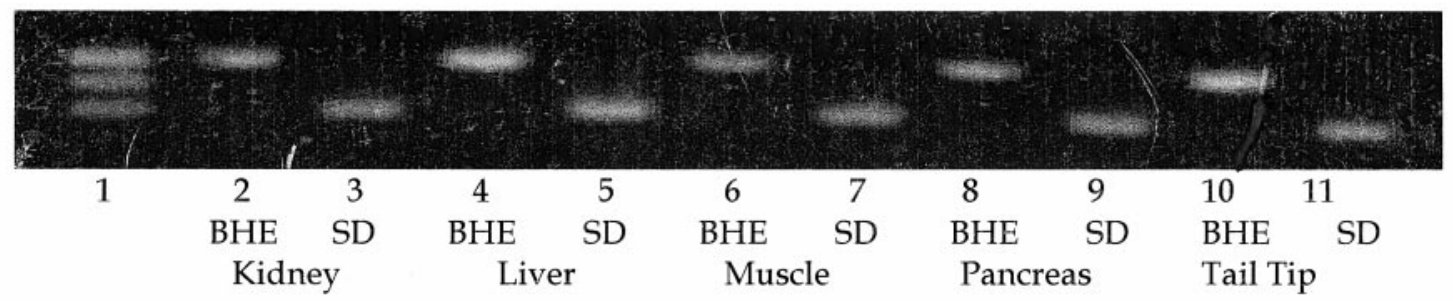




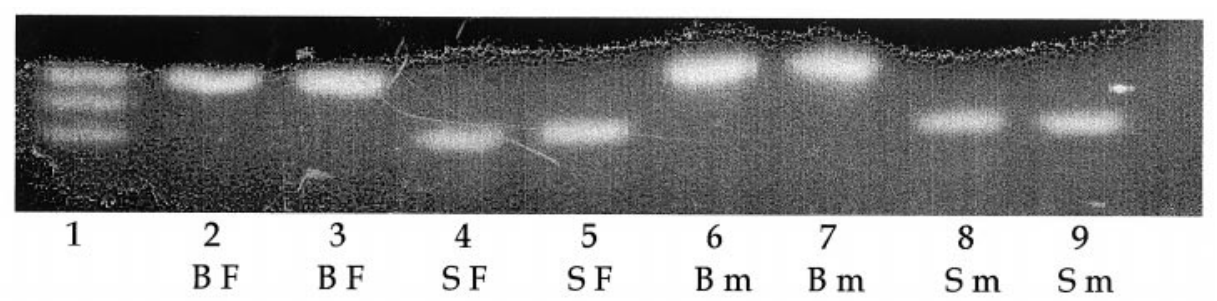

Fig. 2. Determination of the genotype of the $\mathrm{P}$ generation rats. Ladder is in lane 1: bands are 93,100 and 113 base pairs and is a 1:1 mix of the $\mathrm{BHE} / \mathrm{Cdb}$ and SD PCR products. $\mathrm{BF}=\mathrm{PCR}$ products of $\mathrm{BHE} / \mathrm{Cdb}$ females. $\mathrm{Bm}=\mathrm{PCR}$ products of $\mathrm{BHE} /$ $\mathrm{Cdb}$ males. $\mathrm{SF}=\mathrm{PCR}$ products of Sprague Dawley females. $\mathrm{Sm}=\mathrm{PCR}$ products of Sprague Dawley males

ates with the age related impaired glucose tolerance found in $\mathrm{BHE} / \mathrm{Cdb}$ rats. This is the first instance where a mitochondrial mutation in a rat has been shown to lead to impaired glucose tolerance. Unlike humans with mitochondrial diabetes this rat is homoplasmic for its mitochondrial trait.

Many groups have reported mutations and deletions in human mtDNA, which associate with maternally inherited diabetes mellitus [12-19]. Our results show that the BHE/Cdb rat can be used to model these humans. In these rats glucose tolerance is impaired in animals that have a mutated gene for the ATPase subunit 6. Glucose intolerance was maternally inherited as was the ATPase 6 gene sequence and, as expected, cousins that did not carry the mutation had normal glucose tolerance.

How can this defect in mitochondrial DNA explain the impaired glucose tolerance found in the $\mathrm{BHE} / \mathrm{Cdb}$ rat? Insulin secretion is ATP dependent. Requirements for the secretion of insulin include the

Fig. 3. Genotyping the $F_{1}$ generation. Ladder is in lane 1: bands are 93, 100 and 113 base pairs and is a 1:1 mix of SD and $\mathrm{BHE} / \mathrm{Cdb} \mathrm{PCR}$ products. $\mathrm{BF}=\mathrm{PCR}$ products of $\mathrm{BHE} /$ $\mathrm{Cdb}$ females. $\mathrm{Bm}=\mathrm{PCR}$ products of $\mathrm{BHE} / \mathrm{Cdb}$ males. $\mathrm{BXF}=$ PCR products of female cross bred rats with the $\mathrm{BHE} / \mathrm{Cdb}$ dams and $\mathrm{SD}$ fathers. $\mathrm{BXm}=\mathrm{PCR}$ products of male cross bred rats with the BHE/Cdb dams and SD fathers. SXF $=$ PCR products of female cross bred rats with the SD dams and BHE/ $\mathrm{Cdb}$ fathers. $\mathrm{SXm}=\mathrm{PCR}$ products of male cross bred rats with the $\mathrm{SD}$ dams and $\mathrm{BHE} / \mathrm{Cdb}$ fathers. $\mathrm{SF}=\mathrm{PCR}$ products of Sprague Dawley females. $\mathrm{Sm}=\mathrm{PCR}$ products of Sprague Dawley males phosphorylation of glucose via the ATP dependent glucokinase, the need for ATP for the $\mathrm{Ca}, \mathrm{Na}$, and $\mathrm{K}$ exchange that is part of the release mechanism and a high cytosolic ATP:ADP ratio that facilitates this release [20-22]. In addition, the synthesis of insulin (as is the case for all protein synthesis) is ATP dependent. Thus, altogether, any shortfall in the synthesis of ATP, perhaps due to an error in the mitochondrial genome, could have effects on the synthesis and release of insulin. The impaired glucose tolerance shown here is only an early symptom of the diabetic state that will develop later in life. As these rats age their glucose tolerance becomes further impaired and these rats have many of the same clinical features as humans with this type of diabetes [23]. The rats are not obese but do have a fatty liver, they develop severe renal disease and their blood lipids rise with age. These features are diet responsive [1, 3, 4, 23]. In addition, we have already reported on the deterioration in insulin release by isolated islets as these rats age [6] as well as the influence of diet on this age progression $[1,24,25]$. The expression of the diabetes phenotype in humans likewise is subject to dietary influence. Due to the variability of environmental influences that act upon humans, an animal model to study this disease should be attractive. It would appear that the $\mathrm{BHE} / \mathrm{Cdb}$ rat is the first rat model to be reported with a mitochondrial disease and mitochondrial diabetes (mt diabetes), thus, it should be quite useful.

In addition to the finding of the maternal inheritance of both genotype and phenotype, we also report the development of a unique quick and reliable screening test that distinguishes the mutant from the wild type strain of rat. This type of test might have application to human screening projects because of its ease of use and its economy. Other techniques such as direct DNA sequencing, denaturing gradient gel electrophoresis (DGGE), PCR followed by allele specific DNA hybridization or ligation, ribonuclease or chemical cleavage of mismatched heteroduplexes

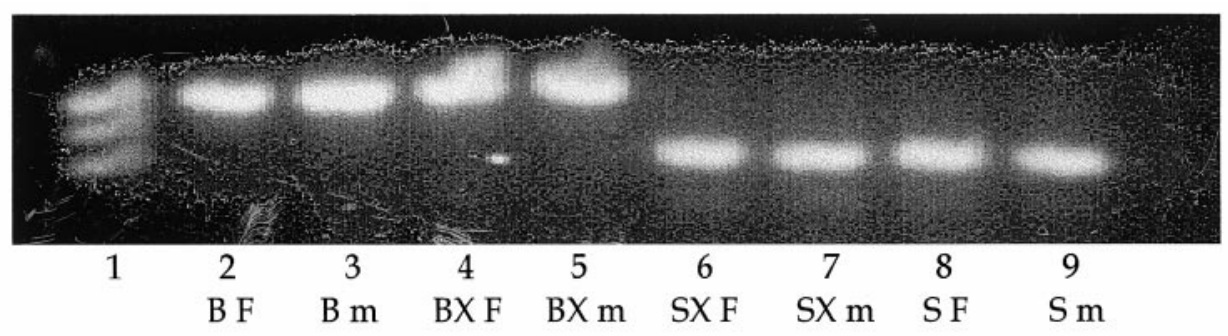


Table 3. Glucose tolerance and fasting blood glucose levels at 100 and 300 days of age in rats not subjected to dietary stress

\begin{tabular}{lllll}
\hline Strain & BHE/Cdb & Bx $^{1}$ & Sx $^{1}$ & Sprague Dawley \\
\hline$n$ & 31 & 29 & 35 & 19
\end{tabular}

100 days of age, males and females

Glucose Tolerance

Area Under Curve $\left(\mathrm{mmol} \cdot \mathrm{min}^{-1} \cdot 1^{-1}\right)$

Fasting Glucose (mmol/l)

$1040.4 \pm 23.1^{\mathrm{a} 2}$

$5.44 \pm 0.18^{\mathrm{z}}$
$1063.9 \pm 18.9^{\mathrm{a}}$
$6.00 \pm 0.22^{\mathrm{x}}$
$963.7 \pm 21.5^{\mathrm{b}}$ $6.05 \pm 0.14^{\mathrm{x}}$ $972.4+21.5^{\mathrm{b}}$ $5.43 \pm 0.10^{z}$

\begin{tabular}{lccc}
\hline$n$ & 10 & 10 & 10 \\
\hline 300 days of age, males only & & & 10 \\
Glucose Tolerance & & & \\
Area Under Curve $\left(\mathrm{mmol} \cdot \mathrm{min}^{-1} \cdot \mathrm{l}^{-1}\right)$ & $1717.2 \pm 20.4^{\mathrm{a}}$ & $1747.0 \pm 24.8^{\mathrm{a}}$ & $1309.6 \pm 20.0^{\mathrm{b}}$ \\
Fasting Glucose $(\mathrm{mmol} / \mathrm{l})$ & $5.56 \pm 0.20$ & $5.88 \pm 0.31$ & $5.01 \pm 0.19$ \\
\hline${ }^{1}$ Cross bred animals: Bx, progeny of female BHE/Cdb and & ${ }^{2}$ Means \pm SEM; means having unlike letter superscripts are \\
Sprague Dawley male; Sx, progeny of female Sprague Dawley & significantly different $(p<0.05)$, as determined by a one way \\
and BHE/Cdb male. & ANOVA followed by Fisher's least significant difference \\
& (LSD) to determine $p$ values.
\end{tabular}
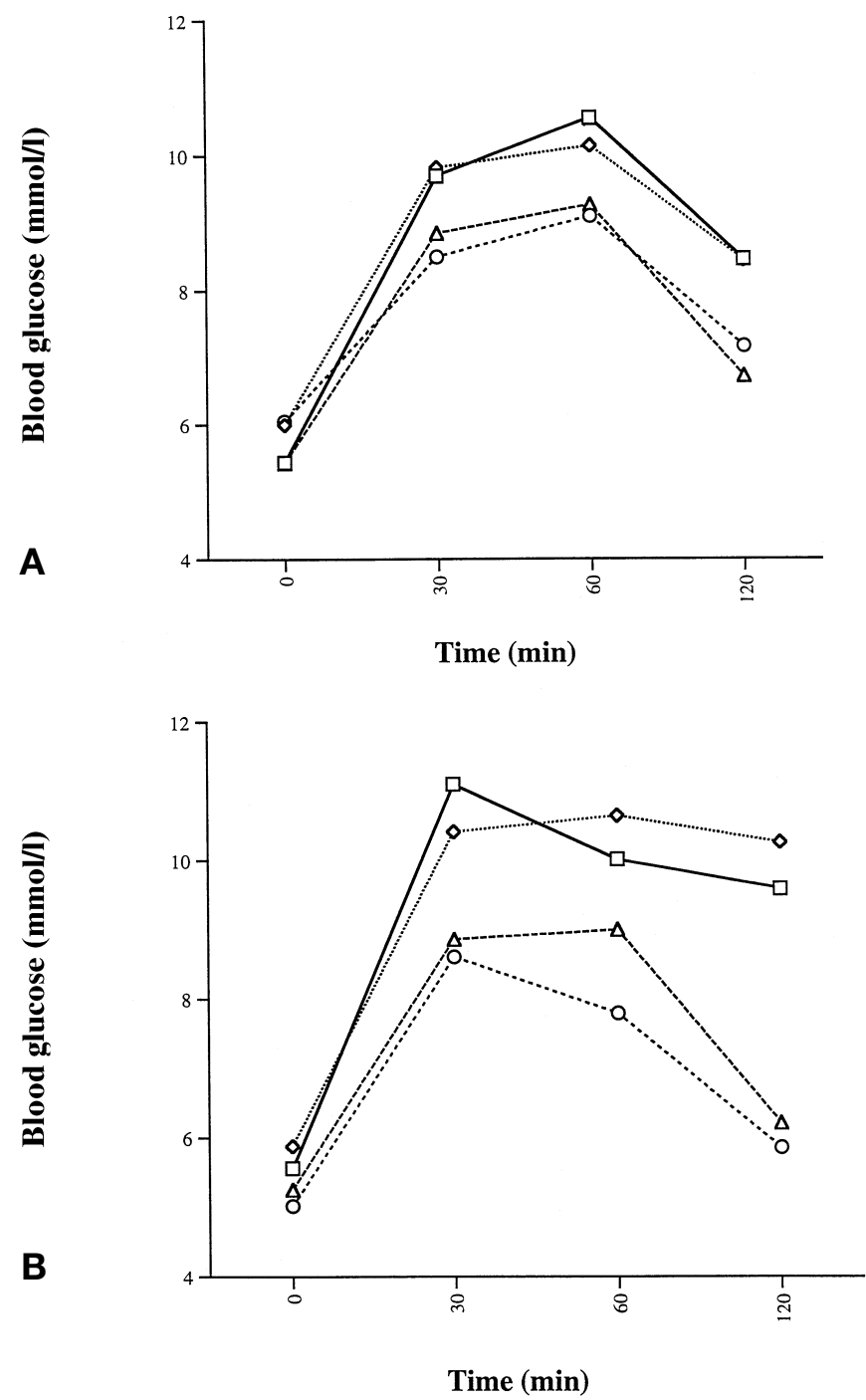

Fig. 4 A, B. Glucose tolerance curve for $\mathrm{BHE} / \mathrm{Cdb}$, Sprague Dawley and Cross Bred Rats. Animals were tested for their glucose tolerance at 100 (A) and 300 (B) days of age. The production of the cross bred rats is described in Table 2.

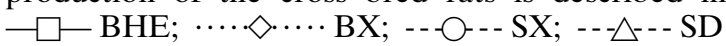

or RFLP are all techniques that have been and are being used to detect point mutations in DNA. These techniques are less likely to come into widespread use in population screening because they are not cost effective, they lack speed or they are not technically feasible for screening purposes. The method described here combines several current ideas of PCR based gene screens. The technique uses a mismatch at the 3 ' end of the PCR primer as described by several investigators [8-11] and primers of different lengths. This addition of competetive primers of different lengths adds to the discrimination. The resultant screening method could thus detect differences in base sequence at a designated site. The method is very useful in screening the BHE/Cdb colony as a quality control measure.

In summary, we have shown that the $\mathrm{BHE} / \mathrm{Cdb}$ can be screened easily for its mutation and that its mutation is clearly linked to its age related impaired glucose tolerance.

Acknowledgements. Supported by the University of Georgia Agricultural Experiment Station project H748, The Bly Fund and the UGA Diabetes Research Fund.

\section{References}

1. Berdanier CD (1994) Non-insulin-dependent diabetes in the BHE/Cdb rat. In: Shafrir E (ed) Lessons From Animal Diabetes. Smith-Gordon, London, pp 231-246

2. Mathews CE, McGraw, RA, Berdanier CD (1995) A point mutation in the mitochondrial DNA of diabetes-prone BHE/Cdb rats. FASEB J 9: 1638-1642

3. McCusker RH, Deaver OE, Berdanier CD (1983) Effect of sucrose or starch feeding on hepatic mitochondrial activity of BHE and Wistar rats. J Nutr 113: 1327-1334

4. Deaver OE, Wander RC, McCusker RH, Berdanier CD (1986) Diet effects on membrane phospholipid fatty acids and mitochondrial function in BHE rats. J Nutr 116: 1148-1155 
5. Berdanier CD, Thomson AR (1986) Comparative studies on mitochondrial respiration in four strains of rats (rattus novegicus). Comp Biochem Physiol 85B:531-535

6. Liang Y, Bonner-Weir, Wu Y-J et al. (1994) In situ glucose uptake and glucokinase activity of pancreatic islets in diabetic and obese rodents. J Clin Invest 93: 2473-2481

7. Sambrook J, Fritsch EF, Maniatis T (1989) Molecular Cloning. A Laboratory Manual. 2nd edn, Cold Spring Harbor, New York

8. Cotton RGH (1992) Detection of single base changes in nucleic acid. Adv Genome Biol 1: 253-300

9. Cotton RGH (1997) Mutation Detection. Oxford University Press, New York, pp 137-142

10. Gibbs RA, Nguyen PN, Caskey CT (1989) Detection of single DNA base differences by competitive oligonucleotide priming. Nucleic Acids Res 17: 2437-2448

11. Sommer SS, Cassady JD, Sobell JL, Bottema CDK (1989) A novel method for detecting point mutations or polymorphisms and its applications to population screening for carriers of phenylketonuria. Mayo Clin Proc 64: 1361-1372

12. Kadowaki H, Tobe K, Mori Y et al. (1993) Mitochondrial gene mutation and insulin-deficient type of diabetes mellitus. Lancet 341: 893-894

13. Awata T, Matsumoto T, Iwamoto MA et al. (1993) Japanese case of diabetes mellitus and deafness with mutation in mitochondrial tRNA (leu(UUR)) gene. Lancet 341: 1291-1292

14. Hirai M, Suzuki S, Onoda M et al. (1996) Mitochondrial DNA 3394 mutation in the NADH dehydrogenase subunit 1 associated with non-insulin-dependent diabetes mellitus. Biochem Biophys Res Comm 219: 951-955

15. Gerbitz KD, Gempel K, Brdiczka D (1996) Mitochondria and diabetes. Genetic, biochemical, and clinical implications of the cellular energy circuit. Diabetes 45: 113-116
16. Velho G, Byrne MM, Clement K et al. (1996) Clinical phenotypes, insulin secretion and insulin sensitivity in kindreds with maternally inherited diabetes and deafness due to mitochondrial tRNA (Leu(UUR)) gene mutation. Diabetes 45: 478-487

17. Gebhart SS, Shoffner JM, Koontz D et al. (1996) Insulin resistance associated with maternally inherited diabetes and deafness. Metabolism 45: 526-531

18. Oka Y, Katagiri H, Ishihara T et al. (1996) Beta-cell loss and glucose induced signalling defects in diabetes mellitus caused by mitochondrial tRNA (Leu(UUR)) gene mutation. Diabet Med 13:S98-S102

19. van den Ouweland JMW, Lemkes HHPJ, Ruitenbeck W et al. (1992) Mutation in mitochondrial tRNA ${ }^{\mathrm{Leu}(U U R)}$ gene in a large pedigree with maternally transmitted type II diabetes mellitus and deafness. Nat Genet 1: 368-371

20. Malaisse WJ (1992) Glucose sensing by the pancreatic $\beta$ cell: the mitochondrial part. Int J Biochem Cell Biol 24: 693-701

21. Nichols CG, Shyng SL, Nestorowicz A et al. (1996) Adenosine diphosphate as an intracellular regulator of insulin secretion. Science 272: 1785-1787

22. Matschinsky FM (1996) A lesson in metabolic regulation inspired by the glucokinase sensor paradigm. Diabetes 45 : 223-241

23. Berdanier CD, Kras K, Wickwire K et al. (1997) Progressive glucose intolerance and renal disease in aging rats. Int J Diabetes 5: 27-38

24. Berdanier CD, Kras K, Wickwire K et al. (1998) Whole egg delays the age related impaired glucose tolerance of BHE/ Cdb rats. Proc Soc Exp Biol Med 219: 28-36

25. Berdanier CD, Johnson B, Hartle DK et al. (1992) Lifespan is shortened in $\mathrm{BHE} / \mathrm{Cdb}$ rats fed a diet containing 9\% menhaden oil and $1 \%$ corn oil. J Nutr 122: 1309-1317 